\title{
RISK FACTORS AND OUTCOMES OF NEW ONSET ARRHYTHMIA IN SURGICAL INTENSIVE CARE UNIT: A RETROSPECTIVE STUDY
}

\author{
Sadaf Hanif, Faisal Shamim, Muhammad Sohaib \\ Aga Khan University Hospital, Karachi Pakistan
}

\section{ABSTRACT}

Objective: To assess the frequency of new-onset cardiac arrhythmias among patients admitted in surgical intensive care unit as well as associated risk factors.

Study Design: Retrospective observational study.

Place and Duration of Study: Surgical Intensive Care Unit, Aga Khan University Hospital, Karachi, from Jan 2018 to Dec 2019. Methodology: The medical record numbers of all patients admitted in surgical intensive care unit were obtained from Surgical intensive care unit case log entries and reviewed. Patients`and healthcare providers` identification were kept confidential. Data was analyzed using SPSS version 19.

Results: Only 13/1076 patients included in the study had cardiac arrhythmias during their stay in Surgical intensive care unit. Among all patients with arrhythmias $(n=13)$, atrial fibrillation was the most common type of arrhythmia $7(53.8 \%)$. The study found higher occurrence of arrhythmias among the patients in the age category of 66 years or above $8(4.6 \%)$ and when compared to younger patients with age categories $18-40$ years $2(0.3 \%)$ and $41-65$ years $3(0.3 \%)$ respectively $(p$-value<0.05). Similarly, arrhythmias were higher among patients who had relatively prolong intensive care unit stay and post-operative status as the primary cause of intensive care unit admission.

Conclusion: Occurrence of cardiac arrhythmias is relatively low among patients admitted to surgical intensive care patient population. Patients age (>65 years), prolong intensive care unit stay, post-operative status are positively associated with development of cardiac arrhythmias among critically ill patients admitted in surgical intensive care unit.

Keywords: Critically Ill patient, New-onset cardiac arrhythmias, Occurrence, Prolong intensive care unit, Surgical intensive care unit.

How to Cite This Article: Hanif S, Sahmim F, Sohaib M. Risk Factors and Outcomes of New Onset Arrhythmia in Surgical Intensive Care Unit: A Retrospective Study. Pak Armed Forces Med J 2021; 71(5): 1880-1884. doi: https://doi.org/10.51253/pafmj.v71i5.5903

This is an Open Access article distributed under the terms of the Creative Commons Attribution License (https://creativecommons.org/licenses/by-nc/4.0/), which permits unrestricted use, distribution, and reproduction in any medium, provided the original work is properly cited.

\section{INTRODUCTION}

Arrhythmias are associated with an unfavorable prognosis, increasing the risk of death. ${ }^{1}$ Progressive cardiovascular deterioration plays a central role in the pathogenesis of multiple organ failure (MOF) caused by sepsis. ${ }^{2,3}$ Critically ill patients often have underlying heart disease, are frequently subject to metabolic, ischemic, or neurohormonal stressors, and are therefore particularly at risk for cardiac arrhythmias. ${ }^{4}$ Cardiac arrhythmias in critically ill patients are most like an indicator of severity of pre-existing critical illness. There is evidence of a high incidence of arrhythmias in patients with non-cardiovascular diseases, such as sepsis, chronic obstructive pulmonary disease and acute renal dysfunction. ${ }^{3}$ Management of new onset arrhythmias is undoubtedly one of the major problems in critical care associated with poor patients' prognosis and clinical outcome. ${ }^{5}$ These arrhythmias may result in prolonged intensive care unit (ICU) and hospital stays. ${ }^{6}$

Correspondence: Dr Sadaf Hanif, Department of Medicine, Aga Khan University Hospital, Karachi Pakistan

Received: 20 Dec 2020; revision received: 01 Feb 2021; accepted: 04 Feb 2021
Most of clinical data has been based on mixed ICU population investigations and evaluated prevalence of supraventricular tachyarrhythmia (8-3.6\%).7 However, there are few studies on the patients admitted to surgical intensive care unit. Hence, there is limited information regarding the incidence and risk factors associated with arrhythmias among patients admitted in surgical ICU.

This study was conducted with an aim to assess the occurrence of arrhythmias among patients admitted in surgical ICU. We also assessed few of the risk factors, associated with new-onset arrhythmias in the surgical ICU patients and its impact on patient outcome. It also determined association of new-onset arrhythmias with hospital mortality among surgical ICU patients.

\section{METHODOLOGY}

This was a retrospective observational study performed at Aga Khan University Hospital after approval from department research committee and institutional ethical review committee (ERC no. 2020-3462-8410) using medical records of patients admitted in Surgical 
Intensive Care Unit (SICU) admitted between January 2018 to December 2019. The tertiary care hospital has a well-established Health Information Management System (HIMS) that ensures quality of all medical records by following standard procedures which also involve management of electronic database of all in-patients or patients admitted in different clinical care units. Inclusion Criteria: The medical records of patients above 18 years of age who admitted in SICU during the specified period.

Exclusion Criteria: Patients who had a positive medical history of under-lying ischemic heart disease or those with pre-existing rhythm disturbances.

Due to limited number of admis-sions at SICU during the study period, all patients meeting the participant selection criteria were included in the study by using non-probability consecutive sampling technique. The medical record numbers of all patients fulfilling the inclusion criteria were obtained from SICU case log entries and then file records were retrieved and reviewed. Variables were collected on a specially designed data collection form. Besides arrhythmia diagnosis by name, ICD codes were also be used to search SICU admission for last 2 years. Patients, staff and healthcare providers personal identification were kept confidential and were not mentioned or recorded on the data collection form.

All analyses was conducted by using the Statistical package for social science SPSS-19). Descriptive statistics were computed for independent variables such as socio-demographic characteristics. Mean and standard deviation were calculated for continuous variables frequencies were calculated for categorical variables. Differences in proportions were assessed by using the chi-square test or Fisher exact test where appropriate. For contrasts of continuous variables, Independent sample t-test was used to assess the difference of mean values. The $p$-value $\leq 0.05$ was statistically significant.

\section{RESULTS}

A total of 1,106 patients were admitted to the surgical ICU of the hospital, from January 2018 to December 2019. In total 1076, records were reviewed while excluding 30 records with incomplete information or records. All the records were assessed for cardiac arrhythmias and type of arrhythmia. However, only 13 patients were found to have cardiac arrhythmias during their stay in Surgical ICU with an occurrence of 13/ 1076 surgical ICU admissions and a total frequency of $1.2 \%$ of all patients observed or included in the study (Figure).

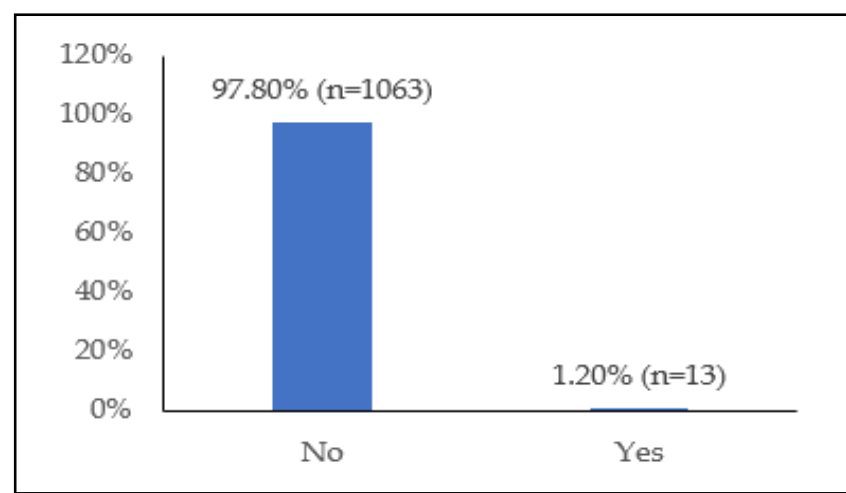

Figure: 24 Months frequency of Cardiac Arrhythmias among Surgical ICU patients admitted with different diagnosis at a private tertiary care hospital in Karachi $(n=1076)$.

Among all patients with arrhythmias $(n=13)$, seven patients had atrial fibrillation, one had atrial flutter, two had paroxysmal SV tachycardia and two had ventricular tachycardia while one patient had second degree heart block (Table-I).

Table-I: Frequency distribution of various types of arrhythmias detected in Surgical ICU patients (in a duration of 24 months) admitted in private tertiary care hospital in Karachi, Pakistan ( $\mathbf{n = 1 3 )}$.

\begin{tabular}{l|c|c}
\hline Type of Arrhythmia & Frequency & Percentage \\
\hline Atrial fibrillation & 7 & 53.8 \\
\hline Atrial flutter & 1 & 7.7 \\
\hline $\begin{array}{l}\text { Paroxysmal } \\
\text { Supraventriculartachycardia }\end{array}$ & 2 & 15.4 \\
\hline Ventricular tachycardia & 2 & 15.4 \\
\hline Second Degree Block & 1 & 7.7 \\
\hline
\end{tabular}

Most of the patients who had arrhythmias only experienced one such event while only one of the patients had four arrhythmia events. The results of our study showed statistically significant differences in the cardiac arrhythmias among the patients with different age categories. It is highest in age group 66 years or above. However, we didn't find any statistically significant difference in the cardiac arrhythmias among patients who had no or one co-morbid as compare to patients who had two or more co-morbids. Similarly, there was no statistically significant difference in the arrhythmias among male compared to female patients. The study also failed to find any statistical significance between common risk factors or comorbid and cardiac arrhythmias (Table-II).

The occurrence of cardiac arrhythmias was 3/29 among patients who admitted to surgical ICU with sepsis which was relatively low as compared to patients without sepsis and it was statistically significant $(p$ value $<0.05$ ). The study also found considerably higher 
Table-II: Distribution of cardiac arrhythmias by selected personal characteristics of patients admitted in Surgical ICU of a private tertiary care hospital in Karachi, Pakistan $(n=1076)$.

\begin{tabular}{|c|c|c|c|c|}
\hline Parameters & Total & $\begin{array}{l}\text { Arrhy- } \\
\text { thmias }\end{array}$ & $\begin{array}{c}\text { No } \\
\text { Arrhythmias }\end{array}$ & $\begin{array}{c}p- \\
\text { value* }\end{array}$ \\
\hline $\begin{array}{l}\text { Age, median } \\
\text { (IQR) } 45 \text { (28) }\end{array}$ & $\mathrm{n}$ & $\mathrm{n}(\%)$ & $\mathrm{n}(\%)$ & \\
\hline \multicolumn{5}{|l|}{ Age Group } \\
\hline $18-40$ years & 447 & $02(0.4)$ & 445 (99.6) & \multirow{3}{*}{$<0.001$} \\
\hline $41-65$ yaers & 456 & $03(0.7)$ & 453 (99.3) & \\
\hline$\geq 66$ & 173 & $08(4.6)$ & $165(95.4)$ & \\
\hline \multicolumn{5}{|l|}{ Gender } \\
\hline Male & 739 & $07(0.9)$ & $732(99.1)$ & \multirow{2}{*}{0.24} \\
\hline Female & 337 & $06(1.8)$ & 331 (98.2) & \\
\hline \multicolumn{5}{|l|}{ Co-morbids } \\
\hline $\begin{array}{l}\text { No or one } \\
\text { co-morbid }\end{array}$ & 856 & 11 (1.3) & 845 (98.7) & \multirow{3}{*}{0.48} \\
\hline Two or more & 000 & $11(1.0)$ & ) & \\
\hline co-morbid & 220 & $02(0.9)$ & $218(99.1)$ & \\
\hline \multicolumn{5}{|l|}{ Hypertension } \\
\hline Yes & 292 & $07(2.4)$ & $285(97.6)$ & \multirow{2}{*}{0.52} \\
\hline No & 784 & $06(0.8)$ & 778 (99.2) & \\
\hline \multicolumn{5}{|c|}{ Diabetes Mellitus } \\
\hline Yes & 240 & $1(0.4)$ & $239(99.6)$ & \multirow{2}{*}{0.31} \\
\hline No & 836 & $12(1.4)$ & $824(98.6)$ & \\
\hline \multicolumn{5}{|c|}{ Chronic Obstructive Airway disease } \\
\hline Yes & 18 & - & $18(100)$ & \multirow{2}{*}{1.00} \\
\hline No & 1058 & $13(1.2)$ & $1045(98.8)$ & \\
\hline \multicolumn{5}{|c|}{ Chronic Kidney Disease } \\
\hline Yes & 24 & - & $24(100)$ & \multirow{2}{*}{1.00} \\
\hline No & 1052 & $13(1.2)$ & $1039(98.8)$ & \\
\hline \multicolumn{5}{|c|}{ Chronic Liver Disease } \\
\hline Yes & 16 & $01(6.3)$ & $15(93.8)$ & \multirow{2}{*}{0.17} \\
\hline No & 1060 & $12(1.1)$ & $1048(98.9)$ & \\
\hline
\end{tabular}

cardiac arrhythmias among patients admitted after undergoing a surgical procedure or in post-operative phase $(9 / 16)$ ( $p$-value $<0.05)$. The study didn't find any association between cardiac arrhythmias and primary diagnosis of trauma, cerebrovascular events, acute respiratory failure and acute kidney injury (Table-III).

The length of stay in ICU was also calculated in numbers of days patient did spend in surgical ICU. Mean length of ICU stay was higher among patients who had arrhythmias (7 days \pm 2.5 ) as compared to patients who had no arrhythmias (4 days \pm 0.9$)$ ( $p$ value $=0.001$ ) (Table-IV). The study didn't find any association between male gender and length of ICU stay, ICU mortality, hospital mortality and number of days on ventilator ( $p$-value $>0.05)$. However, ICU mortality was higher among patients with arrhythmias who had longer ICU stay as compared to patients with arrhythmias with relatively shorter ICU stay.
Table-III: Distribution of cardiac arrhythmias by primary diagnosis or reason of ICU stay among patients admitted in Surgical ICU of a private tertiary care hospital in Karachi, Pakistan (n=1076).

\begin{tabular}{|c|c|c|c|}
\hline $\begin{array}{l}\text { Primary } \\
\text { Diagnosis }\end{array}$ & Arthymias & $\begin{array}{c}\text { No } \\
\text { Arrhythmias }\end{array}$ & $\begin{array}{c}p- \\
\text { value }\end{array}$ \\
\hline Daignosis & Frequency $(\%)$ & Frequency $(\%)$ & \\
\hline \multicolumn{4}{|l|}{ Sepsis } \\
\hline Yes & $03(10.3)$ & $26(89.7)$ & \multirow{2}{*}{0.004} \\
\hline No & $10(89.7)$ & $1037(99)$ & \\
\hline \multicolumn{4}{|c|}{ Post-operative } \\
\hline Yes & 09 (56.3) & $07(43.8)$ & \multirow{2}{*}{$<0.001$} \\
\hline No & $04(0.4)$ & $1056(99.6)$ & \\
\hline \multicolumn{4}{|l|}{ Trauma } \\
\hline Yes & $01(0.5)$ & $193(99.5)$ & \multirow{2}{*}{0.28} \\
\hline No & $12(1.4)$ & $870(98.6)$ & \\
\hline \multicolumn{4}{|c|}{ CNS event / Cardiovascular Accident } \\
\hline Yes & $1(1.1)$ & $93(98.9)$ & \multirow{2}{*}{1.00} \\
\hline No & $12(1.2)$ & $970(98.8)$ & \\
\hline \multicolumn{4}{|c|}{ Acute Respiratory Failure } \\
\hline Yes & - & - & \multirow[b]{2}{*}{-} \\
\hline No & $13(1.2)$ & $1063(98.8)$ & \\
\hline \multicolumn{4}{|c|}{ Acute Kidney Injury } \\
\hline Yes & $1(8.3)$ & $11(91.7)$ & \multirow{2}{*}{0.13} \\
\hline No & $12(1.1)$ & $1052(98.9)$ & \\
\hline
\end{tabular}

${ }^{*}$ Fischer Exact test. $p$-value of $\leq 0.05$ was considered significant Table-IV: Comparison of Length of ICU Stay (Mean number of days) among patients with and without arrthymia $(n=1076)$

\begin{tabular}{l|c|c|c}
\hline Parameter & Arrhythmias & $\begin{array}{c}\text { No- } \\
\text { Arrhythmias }\end{array}$ & $\begin{array}{c}p- \\
\text { value }\end{array}$ \\
\hline $\begin{array}{l}\text { ICU stay in days } \\
\text { (mean } \pm \text { SD) }\end{array}$ & $7 \pm 2.5$ & $4 \pm 0.9$ & 0.001 \\
\hline
\end{tabular}

\section{DISCUSSION}

In this single center surgical intensive care unit study, we found an $1.2 \%$ for all type of cardiac arrhythmias while atrial fibrillation was the commonest type of arrhythmia experienced by majority of the patients. 9,10 The frequency reported in our study is comparatively low as compared to studies conducted among ICU patients previously however, the most common arrhythmias were atrial fibrillation which is in line with previous studies.7-10 This difference in estimates can be explained by the fact that our study was only included patients from surgical ICU while previous studies are mainly conducted among patients from medical ICU or an ICU having a mix of medical as well as surgical patients.

We also observe a statistically significant differences in the arrhythmias when compared among different age categories. Elderly patients who were above 65 years of age had highest cardiac arrhythmias as compared to younger age groups. This finding is supported by a previous study conducted among non-cardiac ICU 
patients which also reported the significantly higher incidence of cardiac arrhythmias among patients who were above 65 years of age. ${ }^{8}$ Fuchs and his colleagues conducted a retrospective analysis of 215 patients admitted between August 2001 and 2008 in a communitybased teaching hospital medical ICU of a large tertiary medical center in Boston. The study concluded that advanced age is an independent risk factor for comorbids and mortality especially for elderly (OR 1.38, 95\% CI: 1.1-1.5). ${ }^{8}$ This can be further explained by findings from a previous study conducted by Hatch and colleagues. The study identified positive correlation between age and risk of cardiac arrhythmias as well as severity of arrhythmias. It concluded that deregulation of intracellular calcium within the heart changes resulting in electrical instability among elderly and increases chances of cardiac arrhythmias. ${ }^{9}$

Our investigation did not detect significant differences in arrhythmias among male and female patients admitted to Surgical ICU. It also didn't find any statistically significant difference in the arrhythmias among patients having no or only one co-morbid when compared to patients with two or more co-morbids. Similarly, we didn't found any statistically significant association between common comorbid conditions and arrhythmias. These findings are contrary to 5 previous and can be explained by relatively smaller sample size and lack of adjustment for potential covariates.1,10,11

Our results did not revealed any statistically significant association between cardiac arrhythmias and diagnosis of sepsis. Surgical ICU patients with sepsis, compared to non-septic patients had lower cardiac arrhythmias. This finding is contrary to previous evidence identifying sepsis as an independent risk factor for cardiac arrhythmias among critically ill patients admitted in ICU. ${ }^{2-16}$ A review of evidence by Schwartz et al also concludes that cardiac arrhythmias sepsis results in complications and increased mortality in ICU patients. ${ }^{12}$ Similarly, a study conducted by Arora in 2006 included 61 patients found that sepsis at admission (9/18 vs. $9 / 43, p=0.01$ ) was associated with high mortality among patients with Atrial Fibrillation. ${ }^{14}$

This can be explained by the fact that septic patients are usually admitted to medical IC $U$ hence resulting in low number of cases among patients admitted to surgical ICU. Cardiac arrhythmias were also associated with a diagnosis of post-operative state or when primary reason of ICU admission was surgery. This finding is supported by previous studies conducted among ICU patients. ${ }^{11,17,18}$
Another finding is a significant association between length of ICU stay and cardiac arrhythmias. Prolong ICU stay has been identified as an independent risk factor for cardiac arrhythmias as previously reported by the studies conducted among patients admitted in medical and cardiology ICU.19-20 They have reported high mortality among patients with arrhythmias who had a longer ICU stay. However, this study didn't take in account the duration of total hospital stays including pre- and post-ICU stay. Hence, we didn't analyze possible association of arrhythmias with total length of hospital duration. Nevertheless, this analysis is a unique addition as contributing to severely scarce evidence regarding arrhythmias among patients admitted to surgical ICUs. The findings from this study can be utilized to identify the potential patients admitted in surgical ICUs who are at high risk of cardiac arrhythmias.

\section{LIMITATION OF STUDY}

Our study had few inherent limitations. First, this was a single-center study focusing surgical patients in ICU only which limits its generalizability to other local settings. Secondly, the medical records didn't provide the information regarding many socio-demographic and medical management related variables such as education, marital status, history of substance abuse, levels of electrolytes and medical investigation. Hence, this ultimately compromised study scope and its utility. Multivariate analysis was not possible due to lack of information about potential confounders. Moreover, the study had a relatively smaller sample size and represents a particular patient population coming to a private tertiary care hospital and admitted in Surgical ICU. Furthermore, due to low arrhythmias a stratified analysis for different types of arrhythmias was not possible.

\section{CONCLUSION}

Our findings suggest a relatively low frequency of cardiac arrhythmias in surgical intensive care patient population. This also concludes that risk factors such as advanced age ( $>65$ years), prolong ICU stay, primary diagnosis of sepsis and post-operative status are associated with development of cardiac arrhythmias among critically ill patients admitted in surgical ICU. However, a more advanced analysis is required to measure the magnitude of the risk and identify more factors while adjusting for key or potential confounders.

\section{Conflict of Interest: None.}

\section{Authors' Contribution}

SH: Idea conceived, data collection, manuscript writing, FS: Development of performa, protocol and proof reading, MS: Development of protocol and proof reading.

\section{REFERENCES}

1. Valderrabano RJ, Blanco A, Santiago-Rodriguez EJ, Miranda C, Del-Rio JRDR, Ruiz J, et al. Risk factors and clinical outcomes of arrhythmias in the medical intensive care unit. J Intensive Care 2016; 4(1): 9-12. 


\section{New Onset Arrhythmia}

2. Walkey AJ, Evans SR, Winter MR, Benjamin EJ. Practice patterns and outcomes of treatments for atrial fibrillation during sepsis: a propensity-matched cohort study. Chest 2016; 149(1): 74-83.

3. Seemann A, Boissier F, Razazi K, Carteaux G, de Prost N, BrunBuisson C, et al. New-onset supraventricular arrhythmia during septic shock: prevalence, risk factors and prognosis. Ann Intensive Care 2015; 5(1): 27-31.

4. Trohman RG, Parrillo JE. Arrhythmias: perspective for the critical care physician. Crit Care Med 2000; 28(10): N115.

5. Goodman S, Weiss Y, Weissman C. Update on cardiac arrhythmias in the ICU. Curr Opin Crit Care 2008; 14(5): 549-554.

6. Goodman S, Shirov T, Weissman C. Supraventricular arrhythmias in intensive care unit patients: short and long-term consequences. Anesth Analg 2007; 104(4): 880-886.

7. Annane D, Sebille V, Duboc D, Le Heuzey JY, Sadoul N, Bouvier E. Incidence and prognosis of sustained arrhythmias in critically ill patients. Am J Respir Crit Care Med 2008; 178(1): 20-25.

8. Fuchs L, Chronaki CE, Park S, Novack V, Baumfeld Y. ICU admission characteristics and mortality rates among elderly and very elderly patients. Intensive Care Med 2012; 38(10): 1654-1661.

9. Makrygiannis SS, Margariti A, Rizikou D, Lampakis M, Vangelis $\mathrm{S}$, Ampartzidou OS, et al. Incidence and predictors of new-onset atrial fibrillation in noncardiac intensive care unit patients. J Crit Care 2014; 29(4): 697-e701.

10. Annane D, Sébille V, Duboc D, Le Heuzey JY, Sadoul N, Bouvier E. Incidence and prognosis of sustained arrhythmias in critically ill patients. Am J Respir Crit Care Med 2008; 178(1): 20-25.

11. Hatch F, Lancaster MK, Jones SA. Aging is a primary risk factor for cardiac arrhythmias: disruption of intracellular Ca2+ regulation as a key suspect. Expert Rev Cardiovasc Therap 2011; 9(8): 1059-1667.
12. Shahreyar M, Fahhoum R, Akinseye O, Bhandari S, Dang G, Khouzam RN. Severe sepsis and cardiac arrhythmias. Ann Transl Med 2018; 6(1): 6-9.

13. Schwartz A, Brotfain E, Koyfman L, Klein M. Cardiac arrhythmias in a septic ICU population: a review. J Crit Care Med 2015; 1(4): 140-146.

14. Arora S, Lang I, Nayyar V, Stachowski E, Ross DL. Atrial fibrillation in a tertiary multidisciplinary intensive care unit: incidence and risk factors. Anaesth Intensive Care 2007; 35(5): 707-713.

15. Werdan K, Schmidt H, Ebelt H, Zorn-Pauly K, Koidl B, Hoke RS, et al. Impaired regulation of cardiac function in sepsis, SIRS, and MODS. Can. J Physiol Pharmacol 2009; 87(4): 266-274.

16. Knotzer H, Mayr A, Ulmer H, Lederer W, Schobersberger W, Mutz N, et al. Tachyarrhythmias in a surgical intensive care unit a case-controlled epidemiologic study. Intens Care Med 2000; 26(7): 908-914.

17. Steinberg JS, Gaur A, Sciacca R, Tan E. New-onset sustained ventricular tachycardia after cardiac surgery. Circulat 1999; 99(7): 903-908

18. Makrygiannis SS, Margariti A, Rizikou D, Lampakis M, Vangelis $\mathrm{S}$, Ampartzidou OS, et al. Incidence and predictors of new-onset atrial fibrillation in noncardiac intensive care unit patients. J Crit Care 2014; 29(4): 697-705.

19. Reinelt P, Karth G, Geppert A, Heinz G. Incidence and type of cardiac arrhythmias in critically ill patients: a single center experience in a medical-cardiological ICU. Intens Care Med 2001; 27(9): 1466-1473.

20. Brathwaite D, Weissman C. The new onset of atrial arrhythmias following major noncardiothoracic surgery is associated with increased mortality. Chest 1998; 114(2): 462-468. 